\title{
A Case Report, Ileo-Ileal Intussusception in Preterm Neonate: An unusual location
}

\author{
Sunil J Pawar ${ }^{1,2 *}$, Shyamsunder $\mathrm{T}^{2}$, Sarveshwar Reddy ${ }^{1}$ and Sahai Chethi ${ }^{1}$ \\ ${ }^{1}$ Department of Pediatrics, Durgabai Deshmukh Hospital and Research Center, India \\ ${ }^{2}$ Thumbay Hospital Hyderabad, India
}

Submission: December 07, 2017; Published: March 19, 2018

*Corresponding author: Sunil J Pawar, Durgabai Deshmukh hospital and research center, Department of Pediatrics Vidya Nagar, Osmania University Road Medical Campus, India, Tel: +919000711682; Email: drsunilpawar1@gmail.com

\begin{abstract}
Background: Intussuception is rare cause of surgical emergency in neonate, and still more rarer in preterm neonate. The clinical features can mimic necrotising enterocoloitis or intestinal obstruction. If left untreated, it can lead to adverse outcomes.

Case summary: We report a case of ileo-ileal intussusceptions in a male preterm infant of 28 weeks of gestation who was born to a primi mother from south India. The neonate initially had feed intolerance, abdominal distention and recurrent apneas on day 8 of life. The x-ray abdomen also showed pneumatosis intestinalis. We kept necrotising enterocolitis as the primary diagnosis. As the neonate deteriorated, surgical exploration was planned. The diagnosis of ileo-ileal intussusceptions was made only at the time of laprotomy. Resection of the gangrenous segment and anastomosis of the healthy gut was done.
\end{abstract}

Conclusion: Intussusception can mimic other abdominal conditions in the neonatal period. Timely suspicion and intervention can improve the outcomes.

Keywords: Intussusceptions; Preterm; Ileo Ileal; Necrotising Enter Colitis

\section{Introduction}

Ileo-ileal Intussusceptions occurrence is very rare in neonatal period and even extremely rare in preterm neonate [1]. The early diagnosis is very important to prevent further complications and adverse outcome. Successful management depends on the early diagnosis and immediate management [2]. We present a case of male preterm neonate presented with clinical features of necrotising enterocolitis and finally diagnosed as ileo-ileal Intussusceptions.

\section{Case Report}

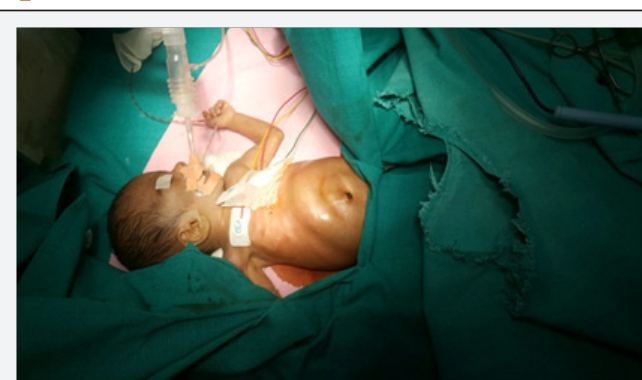

Figure 1: Gross abdominal distension.
A 28 weeks male baby delivered by spontaneous vaginal delivery with birth weight of 1200 grams to primi mother from south India. Neonate cried immediately after birth with an APGAR of 7, 8, 8 at 1, 5 and 10minutes respectively. As the baby was having signs of respiratory distress syndrome started on non-invasive ventilation and received surfactant. Baby started on total parenteral nutrition and colostrum was given from day 1 of life as a unit policy. On day 2, baby started on tube feeding with expressed breast milk. Gradually feeding is increased to full feeds by 6th day of life. On day 8 , baby had recurrent episodes of apnea, poor perfusion and abdominal distension. In view of poor respiratory effort baby connected to ventilator and antibiotics were upgraded as per the protocol. On next day greenish aspirates were noted with tensed abdominal distension (Figure 1). X-ray abdomen showed multiple air fluid level with dilated bowel loops and pneumatosis intestinalis which suggestive of necrotising enterocolitis/intestinal obstruction (Figure 2). The exploratory laparotomy was planned. The intra operative findings were suggestive of ileo-ileal intussusceptions with perforation of the intussuscepted segment (Figure 3). Resection of the necrosed 
segment and reduction of the intussuscepted segment were done. Post operatively, baby required mechanical ventilation for 4 days then weaned to non invasive mode. Gradually feeding started and baby discharged on 84th day of life.

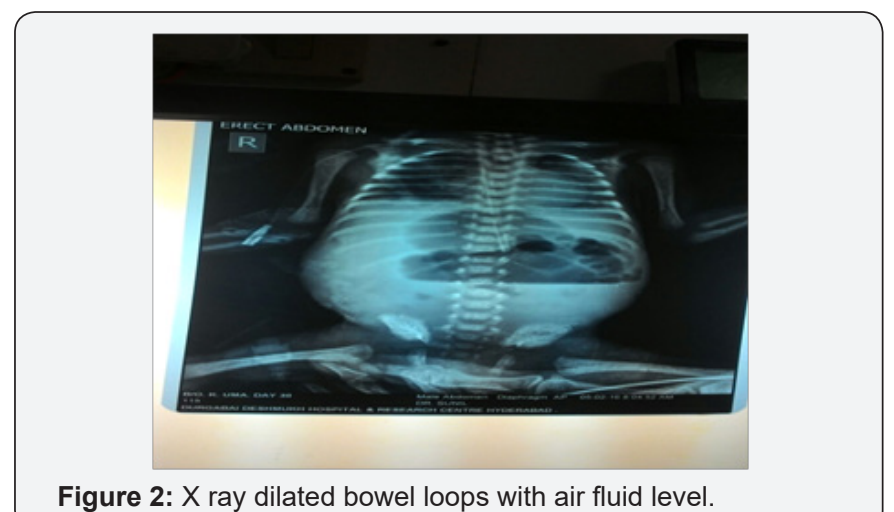

Figure 2: X ray dilated bowel loops with air fluid level.

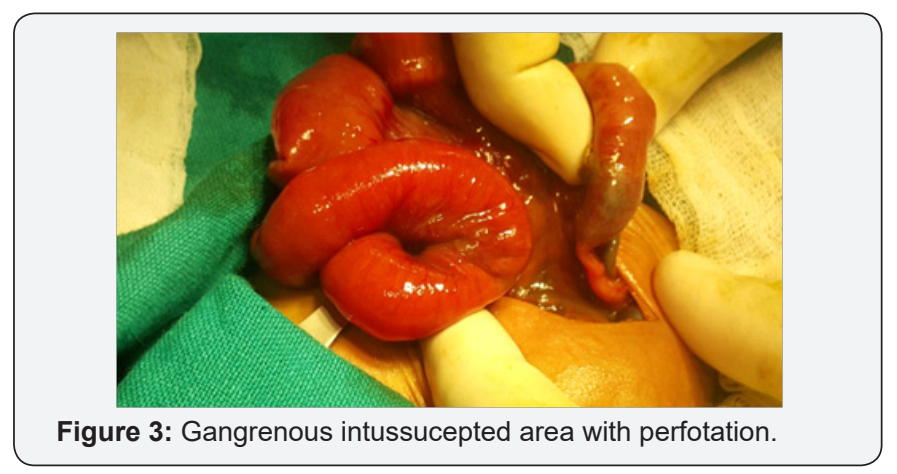

\section{Discussion}

Intussuception is rare in the newborn period, very few cases reported regarding the neonatal intussuception [3]. Intussuception is a process in which a segment of intestine invaginates into the adjoining intestinal segment. The reported incidence of preterm neonate intussuception is $0.3-1.3 \%$ of all cases of intussusceptions [1]. Preterm babies intussuception is still more extremely rare [4]. The most common site for the intussuception is ileum but can occur at other different sites also. Usually abdominal distension, vomiting and bloody stools indicates towards the necrotising enterocolitis but the diagnosis of intussusceptions also be kept in mind to avoid the delay in the treatment. Because the treatment differs from the diagnosis to diagnosis. The early ultrasound of abdomen or contrast enema can help in the early detection of the intussuception but sometimes it's difficult to diagnose if it is associated with the necrotising enterocoloitis and ascites [5].

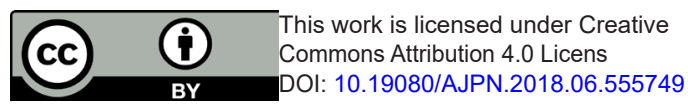

The most common site for the intussuception is ileo colic but it can occur in the other positions like jejuno jejunal, ileo ileal and colio colic [6]. The cause for preterm intussuception still not clear, usually considered as hypoxia or hypo perfusion or some maternal risk factors which act as lead factors. In our case the ultrasound couldn't pick up the intussusceptions may be because of ascites or largely dilated bowel loops. In our case there are also signs of necrotising enterocoloitis, it's difficult to judge that either this is because of intussusceptions or necrotising enterocolitis preceding the intussusceptions.

\section{Conclusion}

The neonate with high index of suspicion with no evidence of sepsis, limited to abdomen and clinically stable can undergo contrast enema or abdominal ultrasonography which is more helpful for early diagnosis and management of the intussusceptions.

\section{Author's contributions}

SJP, SST and SC are the treating team for the neonate. SC have collected the relevant clinical data and formulated the initial draft of case report. SJP and TPO reviewed the literature and corrected the manuscript. SR is the chief surgeon in the surgical team which operated on the neonate. He also looked at the initial manuscript and critically reviewed the same. All the authors have gone through the final manuscript and accepted it.

\section{References}

1. Avansino JR, Bjerke S, Hendrickson M, Stelzner M, Sawin R (2003) Clinical features and treatment outcome of intussusception in premature neonates. J Pediatr Surg 38(12): 1818-1821.

2. JL Grosfeld (2006) Jejunoileal atresia and stenosis. In: Pediatric Surgery, Grosfeld JL, O’Neil JA, Fonkalsrud EW, Coran AG (Eds.), Mosby, Philadelphia, ( $6^{\text {th }}$ edn), USA.

3. Price KJ, Roberton NR, RG Pearse (1993) Intussusception in preterm infants. Arch Dis Child 68(1 Spec No): 41-42.

4. Martínez Biarge M, García Alix A, Luisa del Hoyo M, Alarcón A, Sáenz de Pipaón M, et al. (2004) Intussusception in a preterm neonate; a very rare, major intestinal problem systematic review of cases. J Perinat Med 32(2): 190-194.

5. Ueki I, Nakashima E, Kumagai M, Tananari Y, Kimura A, et al. (2004) Intussusception in neonates: Analysis of 14 Japanese patients. J Paediatr Child Health 40(7): 388-391.

6. Al Salem AH, Hasbash BM (2000) Ileo-ileal intussusception: a report of 4 cases. Ann Saudi Med 20(3-4): 310-312. 